\title{
Propagación de grietas por fatiga de cementos óseos acrílicos. Influencia de los agentes radiopacos
}

\author{
M.P. Ginebra*, L. Albuixech*, E. Fernández-Barragán*, F.J. Gil*, J.A. Planell*, \\ J. San Román** y B. Vázquez**
}

Resumen

En este trabajo se propone el monómero 2,5-diyodo-8-quinolina metacrilato (IHQM) como nuevo agente radiopaco. La adición del agente yodado proporciona un aumento en la resistencia a la tracción, en la tenacidad a la fractura y en la ductilidad con respecto al cemento óseo que contiene sulfato de bario como agente radiopaco. Este efecto se atribuye al hecho de que la utilización del monómero radiopaco elimina la porosidad asociada a las partículas de sulfato de bario que presentan una nula adhesión con la matriz polimérica. Sin embargo, debido a que la resistencia a la propagación de grietas por fatiga es una de las principales propiedades requeridas para el buen comportamiento del cemento en servicio a largo plazo, es importante comparar las propiedades de fatiga de esta nueva formulación de cemento óseo con el cemento que contiene $\mathrm{BaSO}_{4}$. Los resultados muestran que la ausencia de partículas inorgánicas en el cemento juega un papel negativo en la propagación de grietas por fatiga.

Palabras clave Cemento óseo acrílico. Fatiga. Propagación de grietas.

\section{Fatigue crack propagation of acrylic bone cements. Influence of the radio-opaque agents}

\begin{abstract}
In this work the 2,5-diiodo-8-quinolyl methacrylate (IHQM), is proposed as a new radiopaque agent. The addition of the iodine containing methacrylate provided a statistically significant increase in the tensile strength, fracture toughness and ductility, with respect to the barium sulphate containing cement. This effect was attributed to the fact that the use of a radiopaque monomer eliminated the porosity associated to the barium sulphate particles. However, since fatigue resistance is one of the main properties required to ensure a good long-term performance of permanent prostheses, as is the case of acrylic bone cements, it is important to compare the fatigue properties of this new bone cement formulation with the radiolucent and the $\mathrm{BaSO}_{4}$ containing bone cements. The results show that the absence of inorganic particles with no matrix adhesion plays a negative role when the fatigue crack propagation is considered.
\end{abstract}

Keywords Acrylic bone cement. Fatigue. Crack propagation.

\section{INTRODUCCION}

El cemento óseo no actúa solamente como relleno, sino que, además, es el enlace mecánico entre el hueso receptor y la prótesis, favoreciendo un reparto más uniforme de la carga sobre el tejido óseo. El cemento óseo acrílico se obtiene de la mezcla de un líquido incoloro monómero (metacrilato de metilo) y un fino polvo blanco que es el componente polimérico (poli metacrilato de metilo)
(PMMA). Los cementos óseos acrílicos se curan sin temperatura, la reacción se inicia por la acción de una amina terciaria (DMT) sobre el productor de radicales libres: peróxido de benzoilo. Para facilitar el seguimiento del tratamiento se incorporan en los cementos agentes radiopacos para seguir el comportamiento mediante radiografías. En general, como agentes radiopacos se utilizan compuestos inorgánicos, como $\mathrm{BaSO}_{4}$ ó $\mathrm{ZrO}_{2}$, en proporciones que oscilan entre un 8 a un $13 \%$ para el

$\left({ }^{*}\right)$ CREB - Centre de Recerca en Enginyeria Biomèdica, Dpto. Ciencia de los Materiales e Ingeniería Metalúrgica, Av. Diagonal 647, 08028 Barcelona (España).

(**) Instituto de Ciencia y Tecnología de Polímeros. CSIC. Madrid (España). 
sulfato de bario y de un 9 a un $15 \%$ para el óxido de zirconio $^{[1]}$.

Sin embargo, estudios recientes han mostrado que la adición de agentes radiopacos inorgánicos al PMMA produce la diferenciación y proliferación de células osteoclásticas y macrófagos que contribuyen a la reabsorción ósea y al aflojamiento de la prótesis articular ${ }^{[2 \text { y } 3]}$. Asimismo, existen evidencias de que la liberación de partículas de agente radiopaco en los tejidos circundantes pueden causar daños en las superficies articulares, dado el carácter abrasivo de estos agentes, y producir un aumento de residuos de polietileno de ultra alto peso molecular, cuando las partículas se sitúan en el espacio $\operatorname{articular}^{[4-6]}$. Desde el punto de vista del comportamiento mecánico, es bien conocido que la adición de las fases inorgánicas debilita al cemento, ya que no hay adherencia con la matriz polimérica. Las partículas inorgánicas actúan como poros cuando la tensión mecánica es aplicada sobre el cemento ${ }^{[7-}$ 9]. Así, cuando el cemento óseo acrílico contiene un $10 \%$ en peso de $\mathrm{BaSO}_{4}$ se reduce la resistencia mecánica a la tracción ${ }^{[10-12]}$ y la resistencia a flexión en tres puntos ${ }^{[13]}$. Sin embargo, en la influencia del $\mathrm{BaSO}_{4}$ sobre la tenacidad a la fractura, existen resultados controvertidos ${ }^{[14,15]}$.

Teniendo en cuenta las anteriores consideraciones del efecto de los agentes radiopacos inorgánicos, se ha pensado en su sustitución por monómeros radiopacos que polimericen con el cemento óseo. Estos monómeros contienen enlaces con elementos tales como el bromo y/o yodo y pueden copolimerizar en pequeñas cantidades con otros monómeros tales como el monometacrilato de metilo. En este trabajo, se ha utilizado 2,5-diyodo-8-quinolina (IHQM), como nuevo agente radiopaco. En un estudio previo, se constató que el cemento acrílico con IHQM presentaba un adecuado nivel de radiopacidad a partir del $5 \%$ en peso en la fase líquida. Además, la incorporación del agente radiopaco yodado producía un descenso en las temperaturas de polimerización y un ligero aumento en los tiempos de fraguado. En los estudios de implantación en ratas no se apreciaron respuestas inflamatorias crónicas ${ }^{[16]}$. El efecto de la incorporación de este nuevo agente radiopaco sobre algunas propiedades mecánicas fue también objeto de un estudio $^{[15]}$ en el que se constató que producía un aumento, estadísticamente significativo, en la resistencia mecánica, la tenacidad a la fractura y la ductilidad con respecto al sulfato de bario. En este trabajo se aborda el estudio de la resistencia a la propagación de grietas por fatiga del cemento con el nuevo agente radiopaco en relación al cemento convencional sin agente radiopaco.

\section{MATERIALES Y MÉTODO EXPERIMENTAL}

Se han elaborado tres tipos de cementos óseos acrílicos para el estudio de propagación de grietas por fatiga:

- Cemento no radiopaco (sin agente radiopaco): Cemento RL

- Cemento convencional (con sulfato de bario): Cemento BS

- Cemento con agente yodado (con 2,5-diyodo8-quinolina metacrilato): Cemento IHQM

Estos tres cementos se elaboraron con perlas de polimetilmetacrilato (RHOM Plexigum), cuyas características se muestran en la tabla I. Estas fueron mezcladas con MMA o con una mezcla de MMA/IHQM ( $5 \%$ en peso de la fase líquida), dependiendo de la formulación preparada en una relación sólido/líquido de 2:1. El activador utilizado fue la N,N-dimetil-4-toluidina ( $1 \%$ en la fase líquida) y el iniciador de la reacción fue el peróxido de benzoílo ( $2 \%$ en peso de la fase sólida) en todos los casos. Para la preparación del cemento convencional radiopaco (Cemento BS) se añadió a la fase sólida un $10 \%$ del sulfato de bario. El tamaño de las partículas de $\mathrm{BaSO}_{4}$ en el cemento oscilaba entre 0,1 y $3 \mu \mathrm{m}$.

Las probetas CT (Compact Tension) fueron moldeadas al vacío. Se utilizó un molde de teflón para obtener los bloques. Estos fueron posteriormente mecanizados hasta unas dimensiones: $\mathrm{B}=6$ $\mathrm{mm}, \mathrm{W}=23 \mathrm{~mm}$ y a $=11,35 \mathrm{~mm}$, de acuerdo con la norma ASTM E64 ${ }^{[18]}$. El esquema de las probetas utilizadas se ilustra en la figura 1. La entalla se agudizó con una cuchilla de afeitar siguiendo el

Tabla I. Características morfológicas: Diámetro mediano D (50\%), Peso Molecular, tacticidad y contenido de monómero residual para polvo de PMMA utilizado en este estudio

Table I. Morphological characteristics of the PMMA powder: Median diameter D(50\%), Molecular weight, tacticity and residual monomer

\begin{tabular}{|c|c|c|c|c|c|}
\hline & Diámetro & $M_{n} \times 10^{3}$ & $M_{n} / M_{w}$ & cticida & $\begin{array}{c}\text { Monómero } \\
\text { residual }\end{array}$ \\
\hline Perlas de PMMA & 45.06 & 88 & 1,91 & 0,26 & N.d. ${ }^{*}$ \\
\hline
\end{tabular}




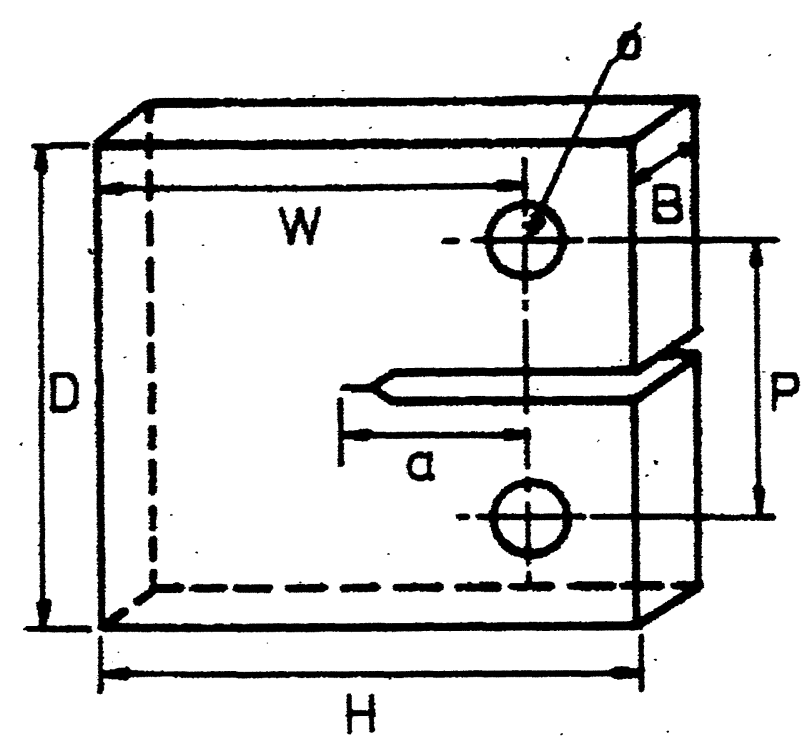

Figura 1. Probeta CT según la norma ASTM E647.

Figure 1. CT specimen according to ASTM E647 standard.

protocolo del Grupo Europeo de Fractura ${ }^{[19]}$. Las muestras, antes de ser ensayadas mecánicamente, se almacenaron durante 1 mes en solución de Ringer's a $37^{\circ} \mathrm{C}$.

Los ensayos de fatiga se realizaron en una máquina servohidráulica (MTS Bionix 858) a temperatura ambiente. Los ensayos se llevaron a cabo bajo control de carga a una frecuencia de $2 \mathrm{~Hz}$ aplicando cargas tracción-tracción con una $R=1 / 3$ y una carga media de $50 \mathrm{~N}$. Las superficies de fractura se observaron mediante microscopía electrónica de barrido. El número de muestras ensayadas para cada formulación de cemento se recoge en la tabla I. La longitud de las grietas se midió mediante una cámara de video CCD 4005R y un sistema de medición $V \mathrm{~ms}$, con una resolución de $1 \mu \mathrm{m}$. Para cada muestra se representaron las curvas de la velocidad de propagación de grieta por ciclo (da/dN) frente a la amplitud del factor de intensidad de tensiones en modo I $\left(\Delta \mathrm{K}_{\mathrm{I}}\right)$ en escala logarítmica, confirmándose que los cementos siguen el modelo de propagación de grietas por fatiga de $\mathrm{Pa}$ ris-Erdogan ${ }^{[20-22]}$ :

$$
\mathrm{da} / \mathrm{dN}=\mathrm{A}\left(\Delta \mathrm{K}_{\mathrm{I}}\right)^{\mathrm{m}}
$$

donde A y m son constantes que dependen del material y de las condiciones ambientales. El parámetro A se obtiene en la intersección de la curva en el eje da/dN cuando $\Delta K_{I}=1$, y m es la pendiente, que se interpreta como una medida del aumento de la velocidad de la propagación de la grieta cuando ésta aumenta su longitud.

\section{RESULTADOS EXPERIMENTALES}

La propagación de grietas por fatiga de los cementos ensayados bajo diferentes condiciones se muestra en la figura 2. En la tabla II se muestra los valores medios de $\mathrm{A}$ y $\mathrm{m}$ de la ecuación de Paris-Erdogan obtenida para las tres diferentes series de muestras. En relación al cemento que contiene sulfato de bario se puede apreciar que los resultados obtenidos se ajustan bastante bien a los referenciados por otros autores en condiciones de ensayo similares a las de este trabajo ${ }^{[22-24]}$.

De acuerdo con los datos obtenidos, no hay diferencias estadísticamente significativas en la propagación de grietas por fatiga del cemento sin agente radiopaco y el que contiene el monómero yodado (IHQM). Sin embargo, el cemento BS muestra una mayor resistencia a la propagación de grietas por fatiga. Así, tanto A como el exponente de creçimiento de grieta $m$ para el cemento BS son menores que los obtenidos para los otros dos cementos estudiados. Este hecho significa que para un rango de intensidad de tensiones dado, el cemento BS tiene una menor velocidad de propagación de grietas y una menor aceleración de la misma en relación con el cemento sin agente radiopaco y a aquél que contiene el agente yodado. Además, para el cemento BS, la propagación

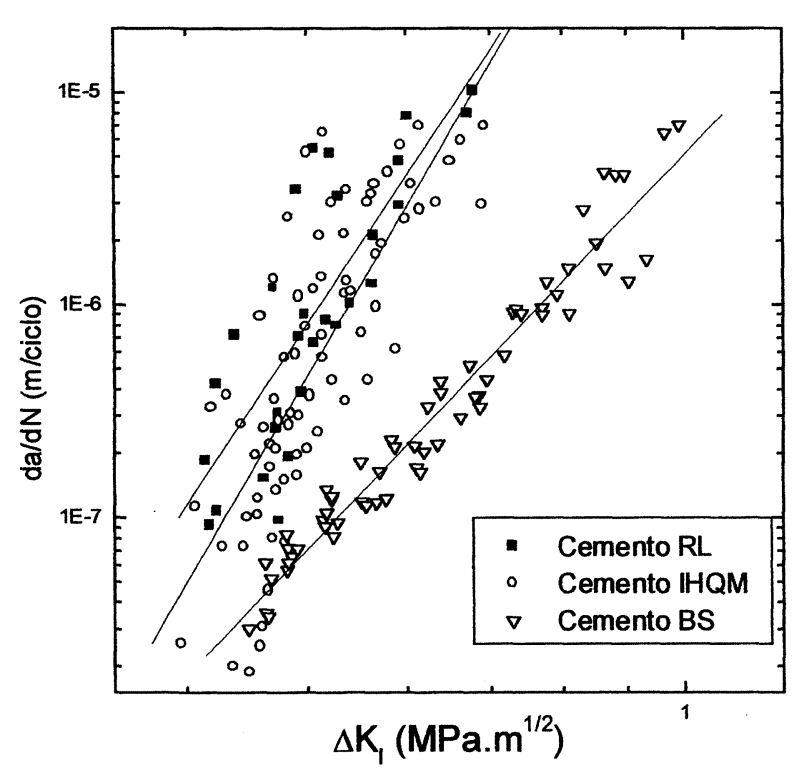

Figura 2. Crecimiento de grietas por fatiga y rectas de regresión ajustadas para los diferentes cementos estudiados.

Figure 2. Fatigue crack growth data and associated regression lines for the different cement studied.

Rev. Metal. Madrid 37 (2001) 
Tabla II. Parámetros de la ecuación de Paris-Erdogan obtenidos para las tres formulaciones de cemento estudiadas

Table II. Paris-Erdogan parameters for the three cement formulations studied

\begin{tabular}{lcccc}
\hline CEMENTO & $\begin{array}{c}\mathbf{N}^{\circ} \text { de } \\
\text { muestras }\end{array}$ & M & A (m/ciclo) & $\begin{array}{c}\text { Coeficiente } \\
\text { de } \\
\text { correlación }\end{array}$ \\
\hline Cemento RL & 4 & 8,8 & $3,63 \times 10^{-4}$ & 0,65 \\
Cemento BS & 8 & 6,15 & $5,09 \times 10^{-6}$ & 0,95 \\
Cemento IHQM & 8 & 10,5 & $4,89 \times 10^{-4}$ & 0,61 \\
\hline
\end{tabular}

de la grieta estable tiene lugar hasta valores más elevados de la amplitud del factor intensidad de tensiones $\left(\Delta \mathrm{K}_{\mathrm{I}} \approx 1 \mathrm{MPa} \cdot \mathrm{m}^{1 / 2}\right)$, respecto a las otras dos formulaciones, en las que el límite de la propagación estable de la grieta aparece para $\Delta \mathrm{K}_{\mathrm{I}} \approx 0,7$ $\mathrm{MPa} \cdot \mathrm{m}^{1 / 2}$. Este comportamiento esta de acuerdo con los resultados encontrados en la literatura para los cementos que contienen sulfato de bario comparados con los que no contienen agentes radiopa$\cos ^{[17 \text { y } 25] \text {. }}$

Estos resultados confirman que la adición de partículas de $\mathrm{BaSO}_{4}$ a la matriz polimérica tiene un efecto beneficioso respecto al comportamiento a fatiga del cemento. La pobre adhesión entre las partículas inorgánicas y la matriz polimérica actúa de forma análoga a como lo harían pequeños poros, dificultando la propagación de grietas por fatiga. Diferentes hipótesis han sido propuestas para explicar este comportamiento. Entre los mecanismos planteados se sugiere que la incorporación de las partículas de $\mathrm{BaSO}_{4}$ produce un debilitamiento de la matriz del cemento, facilitando la propagación a través de la misma y no de las partículas de polvo prepolimerizado, dando lugar a un camino de propagación más largo ${ }^{[17]}$.

Otras teorías apuntan a que este comportamiento podría estar relacionado con la absorción de energía por los poros provocando nuevas microgrietas dando lugar a un camino más sinuoso en la propagación de grietas por fatiga ${ }^{[26]}$. A partir de los estudios fractográficos, se aprecia que para el cemento RL y el cemento IHQM la superficie de fractura es lisa y muestra una propagación de la grieta plana (Figs. 3 y 4). En cambio, en el cemento BS la grieta progresa principalmente a través de la matriz evitando fracturar las perlas de PMMA y por tanto la superficie de fractura es más rugosa (Fig.5).

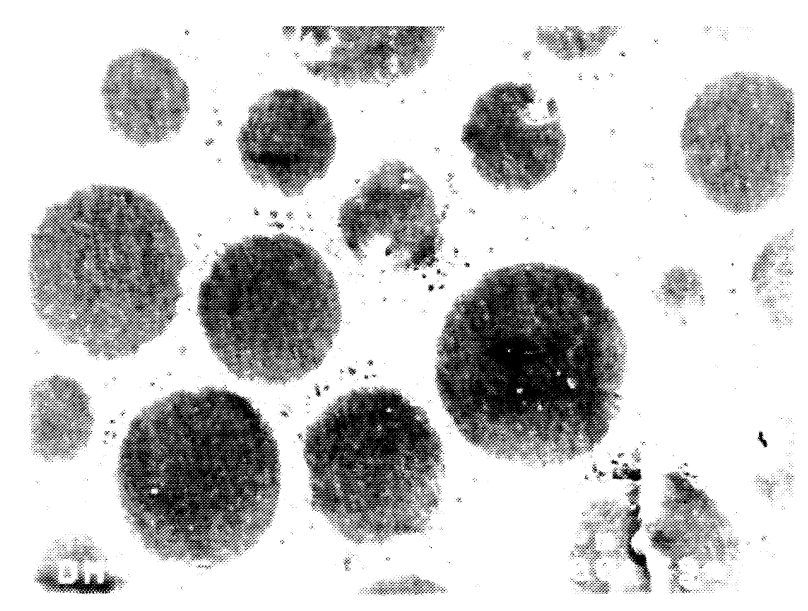

Figura 3. Superficie de fractura cemento RL.

Figure 3. Fracture surface of RL cement.

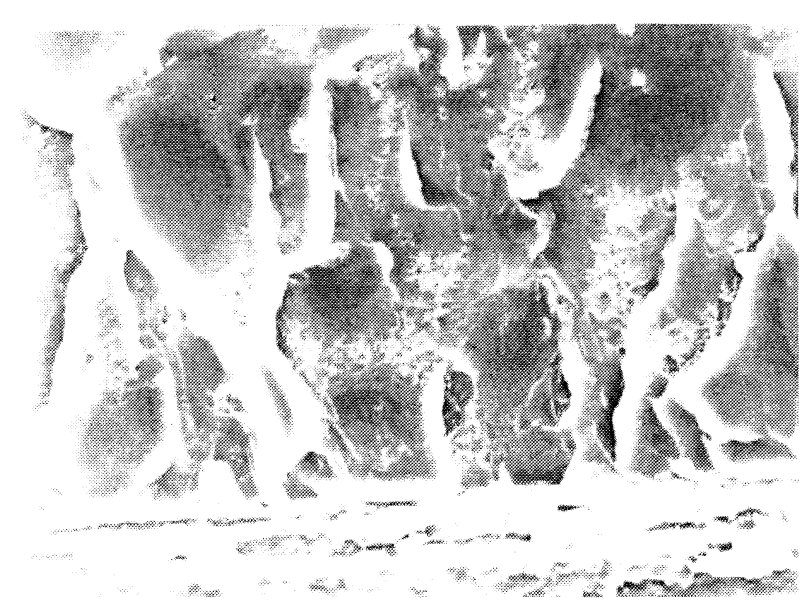

Figura 4. Superficie de fractura cemento IHQM.

Figure 4. Fracture surface of IHQM cement.

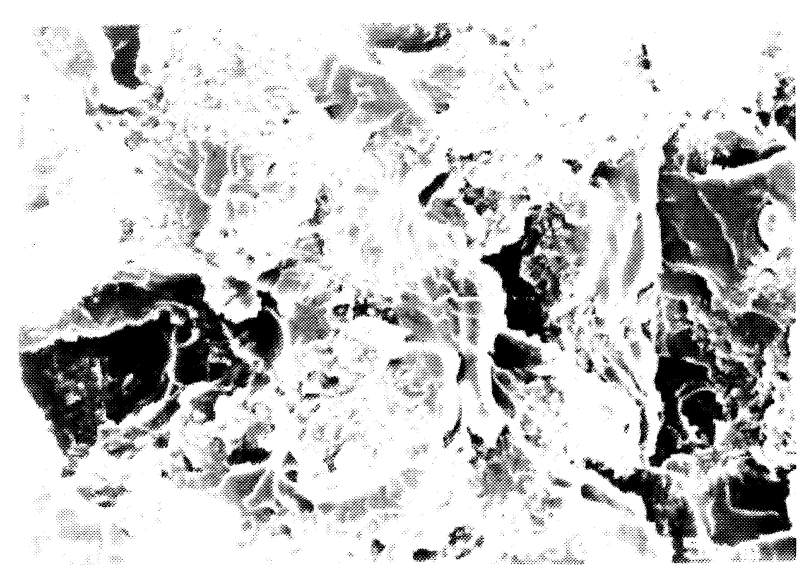

Figura 5. Superficie de fractura cemento BS.

Figure 5. Fracture surface of BS cement. 


\section{Agradecimientos}

Los autores agradecen a la CICYT la ayuda prestada (MAT99-1064)

\section{REFERENCIAS}

[1] K.D. KÜHN, Springer Verlag, Berlin, 2000.

[2] A. Sabokbar, Y. Fujikawa, D. Murray y N. Athanasou, J. Bone Joint Surg. (Br.) 79-B (1997) 129.

[3] M. Lazarus, J. Cuckler, H. Schumacher, P. Ducheyne, D.G. BAKER y J. ORTHOP, Res. 12 (1994) 532.

[4] G.H. Isaac, J.R. AtKinson y D. Dowson et al., Eng. Med. 16(3) (1987) 167.

[5] L. Caravia, D. Dowson, J. Fisher y B. Jobbins, Proc. Inst. Mech. Eng. 204 (1990) 65.

[6] J.R. CoOper, D. Dowson, J. Fisher y B. Jobbins, J. Med.Eng. E Tech. 15 (1991) 63.

[7] L.D.T. TOPOleski, P. DuCheyne y J.M. CuCKLeR, J. Biomed. Mater. Res. 24 (1990) 135.

[8] M. Vila, M.P. Ginebra, J. Gil y J. Planell, J. Biomed. Mater. Res. (Appl. Biomat.) 48 (1999) 128.

[9] A. Owen y P. Beaumont, Mechanical Properties of Biomaterials, G. Hastings and D. Williams, John Wiley\&Sons Ltd (Eds.), 1980, pp. 277.

[10] S.S. HaAs, G.M. Brauer, M.A. Dickson y J. Bone, Joint Surg. 57-A (3) (1975) 380.

[11] R.O. KusY, J.Biomed. Mater.Res. 12 (1978) 271.

[12] B. VÁzQuez, S. Deb y W. Bonfield, J. Mater. Sci. Mater. Med. 8 (1997) 455.

[13] N.J. Holm, Acta Orthop. Scand. 48 (1977) 436.
[14] G.C. Sin y A.T. Berman, J. Biomed. Mater. Res. 14 (1980) 311.

[15] M.P. Ginebra, C. Aparicio, L. Albuixech, E. Fernández-Barragán, F.J. Gil, J.A. Planell, L. Morejón, B. VÁzQUez y J. SAN Román, J. Mater. Sci. Mater. Med. 10 (1999) 733-737.

[16] B. Vázquez, M.P. Ginebra, F.J. Gil, J.A. Planell, A. López Bravo y J. SAN ROMÁn, Biomaterials 20 (1999) 2047-2053.

[17] L.N. Molino y L.D.T. TOPOlESKI, J. Biomed. Mater. Res. 31 (1996) 131-137.

[18] ASTM STD E647, Annual Book of ASTM Standards, Part 3.01, American Society for Testing and Materials, Philadelphia, 1992, p. 645.

[19] J.G. Williams, "Testing protocol, Standard for determining $K_{c}$ and $G_{c}$ for plastics. EGF Newsletter, 8, Winter, 1988/89, pp.14.

[20] R.W. Hertzberg, Deformation and fracture mechanics of engineering materials, $4^{\text {th }}$ John Wiley \& Sons Inc. (eds.), New York, 1996, p. 664

[21] G. Lewis, J. Biomed. Mater. Res. (Appl. Biomater.), 38 (1997) 155-182.

[22] N.C. Nguyen, W.J. Maloney y R.H. Dauskardt, J. Mater. Sci. Mater. Med. 8 (1997) 473-483.

[23] T.M. Wright y R.P. Robinson, J. Mater. Sci. 17 (1982) 2463-2468.

[24] Y.K. Liu, J.B. Park, G.O. Njus y D. Stienstra, J. Biomed. Mater. Res. 21 (1987) 247-261.

[25] P.W.R. Beaumont, J. Biomed. Eng. 1 (1979) 147-152.

[26] J. Lankford, W.J. Astleford y M.A. Asher, J. Mater. Sci. 11 (1976) 1624-1630. 